
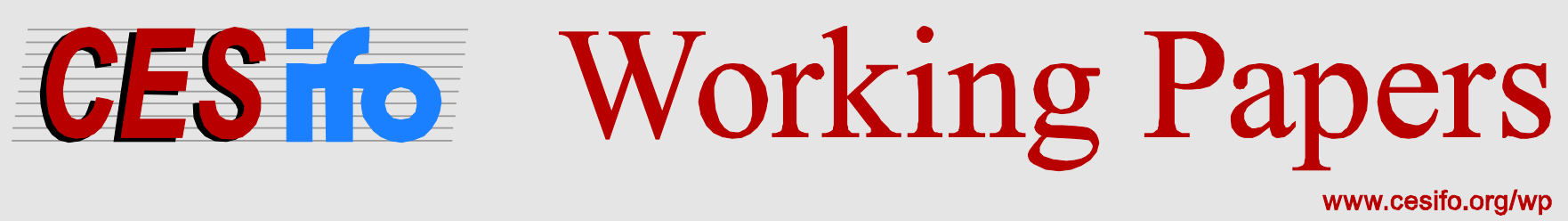

\title{
The Exploitation Hypothesis in a Public Good Economy: Some Extensions
}

\author{
Wolfgang Buchholz \\ Todd Sandler
}

CESIFO WORKING PAPER NO. 5717

CATEgORY 1: PUBlic FinANCE

JANUARY 2016

An electronic version of the paper may be downloaded

- from the SSRN website:

- from the RePEc website:

- from the CESifo website:

www.SSRN.com

Www.RePEc.org

www.CESifo-group.org/wp 


\title{
The Exploitation Hypothesis in a Public Good Economy: Some Extensions
}

\begin{abstract}
Since Olson's (1965) The Logic of Collective Action, the exploitation hypothesis, in which the rich shoulders the provision burden of public goods for the poor, has held sway despite empirical exceptions. To address such exceptions, we establish two alternative exploitation hypotheses based on asymmetric preferences or on productivity differences regarding the public good. The classic hypothesis and its two variants are proven in a novel fashion. Our theoretical insights are then illustrated by some empirical examples from the field of international public goods, such as military defence and cross-border pollution.
\end{abstract}

JEL-codes: F530, H410, H870.

Keywords: public goods, exploitation hypothesis.

Wolfgang Buchholz University of Regensburg Germany - 93040 Regensburg wolfgang.buchholz@ur.de
Todd Sandler

University of Texas at Dallas

USA - Richardson, Texas 75080-3021

tsandler@utdallas.edu 


\section{Introduction}

Beginning with Olson (1965), the "exploitation hypothesis" has been of recurrent interest in the theory of public goods (see, e.g., Sandler, 1992; Cornes and Sandler, 1996; Boadway and Hayashi, 1999; Ihori, McGuire and Nakagawa, 2014). This hypothesis essentially means that, in a Nash equilibrium of voluntary public good provision, the rich (better endowed) agents make larger contributions to the public good than the poor ones so that the rich agents, in a certain sense, are "exploited" via the strategic context of the contribution game. In Sandler (2015, pp. 206-209), it has, however, been remarked that the hypothesis can only be expected to hold under specific circumstances, i.e., if the agents behave according to the strategic Nash assumption and have identical preferences. In this vein, we show that - while keeping the Nash assumption - besides the original "exploitation of the rich by the poor" there are also other kinds of "exploitation", i.e., of the agents with a high preference for the public good by those with a low preference, and of the agents with greater productivity in providing the public good by those with lower productivity.

The structure of our paper is as follows: After describing the framework of the analysis in Section 2, we consider the three variants of the exploitation maxim in Section 3. In particular, we provide a short novel proof of the classical exploitation hypothesis and its two variants there. In Section 4, examples illustrate how the partial effects underlying the three versions of the exploitation hypothesis may oppose each other. In Section 5, we conclude by suggesting some empirical applications of our theoretical results.

\section{The Framework}

There are $n$ agents $i=1, \ldots, n$, who are characterized by their initial private good endowments $w_{i}$ and their utility functions $u_{i}\left(x_{i}, G\right)$, where $x_{i}$ denotes agent $i$ 's level of private consumption and $G$ indicates public good supply. Each utility function is assumed to have the standard properties, i.e., it is twice continuously differentiable, quasiconcave, and strictly monotone increasing in both variables. Moreover, both goods are assumed to be non-inferior for all agents. Given some marginal rate of substitution $m r s_{i}=\alpha_{i}$, each agent i's (income) expansion path $e_{i}\left(G, \alpha_{i}\right)$, derived from her utility function $u_{i}\left(x_{i}, G\right)$, is a well-defined and strictly monotone increasing (and differentiable) function of $G$. In $x_{i}$-G space, such an expansion path connects all points $\left(x_{i}, G\right)$ at 
which agent $i$ 's indifference curves have slope $-\alpha_{i}$ so that $\alpha_{i}=\frac{\partial u_{i} / \partial x_{i}}{\partial u_{i} / \partial G}\left(x_{i}, G\right)$ holds. In order to avoid the tedious treatment of sub-cases, we assume $e_{i}\left(0, \alpha_{i}\right)=0$ and $\lim _{G \rightarrow \infty} e_{i}\left(G, \alpha_{i}\right)=\infty$, which, e.g., results when individual preferences are of the CobbDouglas type.

Agents may also have different constant productivities $a_{i}$ (= individual marginal rates of transformation $m r t_{i}$ ) in providing the public good. These productivities indicate how many units of the public good agent $i$ generates if she spends one unit of the private good on the public good. The reciprocal of the productivity parameter $a_{i}$ then represents agent $i$ 's cost of producing one unit of the public good. Given such an $a_{i}$, we let $\bar{G}_{i}\left(w_{i}, a_{i}\right)$ be the level of public good supply at which the value of the expansion path $e_{i}\left(G, a_{i}\right)$ corresponds to agent $i$ 's initial endowment $w_{i}$, i.e., $e_{i}\left(\bar{G}_{i}\left(w_{i}, a_{i}\right), a_{i}\right)=w_{i}$ holds (later shown in Figure 1). From our assumptions, it follows from the intermediate value theorem that $\bar{G}_{i}\left(w_{i}, a_{i}\right)$ exists, and the monotonicity of the expansion path implies that it is unique (see again Figure 1 for a visualization).

Given initial endowment $w_{i}$, utility functions $u_{i}\left(x_{i}, G\right)$, and productivity parameters $a_{i}$, it well-known that, under the assumptions imposed above, the Nash equilibrium (NE) $\left(\hat{x}_{1}, \ldots, \hat{x}_{n}, \hat{G}\right)$ of voluntary public good provision exists and is unique (Bergstrom, Blume and Varian, 1986; Fraser, 1992; Cornes and Sandler, 1996; Cornes and Hartley, 2007). This NE, in which no agent can make a negative public good contribution, possesses the following properties:

(i) The feasibility constraint $\hat{G}=\sum_{i=1}^{n} a_{i} \hat{z}_{i}$ is satisfied, where $\hat{z}_{i}=w_{i}-\hat{x}_{i} \geq 0$ is agent i's public good contribution.

(ii) The equilibrium position of each contributing agent $i$, i.e., for whom $\hat{z}_{i}>0$ holds, lies on her expansion path $e_{i}\left(G, a_{i}\right)$, i.e., $\hat{x}_{i}=e_{i}\left(\hat{G}, a_{i}\right)$.

(iii) Agent $i$ is a contributor if and only if $\hat{G}<\bar{G}_{i}\left(w_{i}, a_{i}\right)$ (see Andreoni, 1988).

We now make use of the second and the third of these properties to present three different versions of the exploitation hypothesis. 


\section{Three Versions of an Exploitation Hypothesis}

The three versions are obtained by assuming that the agents only differ with respect to endowment levels, or preferences, or productivities, while they are homogenous in terms of the other two properties.

Case 1: All agents have the same utility function $u\left(x_{i}, G\right)$ and the same productivity parameter $a$ but have different income levels $w_{i}$.

The relevant expansion path, which is then common to all agents, is denoted by $e(G, a)$. Without loss of generality, we assume $w_{1} \leq \ldots \leq w_{n}$. The monotonicity of $e(G, a)$ thus gives $\bar{G}_{1}\left(w_{1}, a\right) \leq \ldots \leq \bar{G}_{n}\left(w_{n}, a\right)$. In Figure 1, we, specifically, consider two agents, $j$ and $k$, with $j<k$, for whom $\bar{G}_{k}\left(w_{k}, a\right)>\bar{G}_{j}\left(w_{j}, a\right)>\hat{G}$ holds so that both are contributors to the public good.

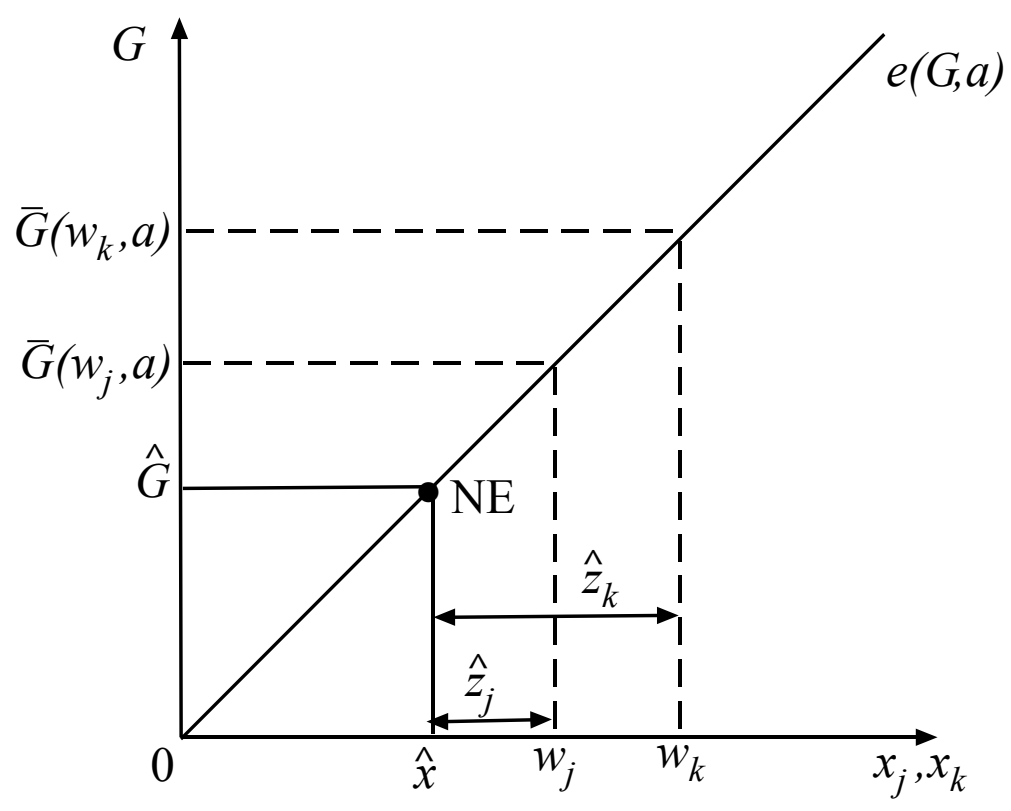

Figure 1. Classical income-based exploitation hypothesis

From property (iii), it follows that, at the NE, there is some $m^{*} \geq 1$ where the group of contributors consists of all rich agents for whom $i \geq m^{*}$, whereas the group of noncontributors consists of poorer agents for whom $i<m^{*}$. Furthermore, property (ii) implies that, at the NE, all contributors have the same private consumption $\hat{x}=e(\hat{G}, a)$. 
Therefore, at the NE, the absolute levels of public good contributions of the contributing agents $\hat{z}_{i}=w_{i}-\hat{x}$ vary positively with income $w_{i}$. This is also seen on the horizontal axis of Figure 1. The same holds true for the relative contribution levels $\frac{\hat{z}_{i}}{w_{i}}=1-\frac{\hat{x}}{w_{i}}$, which establishes Olson's conventional exploitation maxim. Obviously, at the NE, all contributing agents attain the same utility $u(\hat{x}, \hat{G})$, whereas non-contributors attain a smaller utility $u\left(w_{i}, \hat{G}\right)$, ranked by income.

Case 2: All agents have the same income $w$ and the same public good productivity $a$ but possess different utility functions $u_{i}\left(x_{i}, G\right)$.

In particular, we assume that agents can be ordered according to their marginal willingness to pay for the public good. Precisely, at each $(x, G)$, we have $m r s_{j}=\frac{\partial u_{j} / \partial x_{j}}{\partial u_{j} / \partial G}(x, G) \geq$ $\frac{\partial u_{k} / \partial x_{k}}{\partial u_{k} / \partial G}(x, G)=m r s_{k}$ if $j, k \in\{1, \ldots, n\}$ with $j<k$. The indifference curves of an agent with a lower rank $j$ are thus everywhere steeper than the indifference curves of an agent with a higher rank $k$, so that agent $k$ has a greater relative preference for the public good than agent $j$. Convexity of indifference curves then implies that $e_{j}(G, a)$ $\geq e_{k}(G, a)$ for any $G$ if $j<k$ and thus $\bar{G}_{1}(w, a) \leq \ldots \leq \bar{G}_{n}(w, a)$ (see Figure 2).

Property (iii) shows that there is a $m^{*} \geq 1$ for which only agents $i \geq m^{*}$ contribute to the public good, while agents with less interest in the public good than agent $m^{*}$ contribute nothing at the NE. For contributing agents $j$ and $k$, we have, as depicted in Figure 2, that $\hat{x}_{j}=e_{j}(\hat{G}, a) \geq e_{k}(\hat{G}, a)=\hat{x}_{k}$ and hence $\hat{z}_{j}=w-\hat{x}_{j} \leq w-\hat{x}_{k}=\hat{z}_{k}$ if $j<k$. This shows that, ceteris paribus, agents with the greater preference for the public good spend more on it at the NE in absolute and, as income $w$ is the same for all agents here, also in relative terms. 


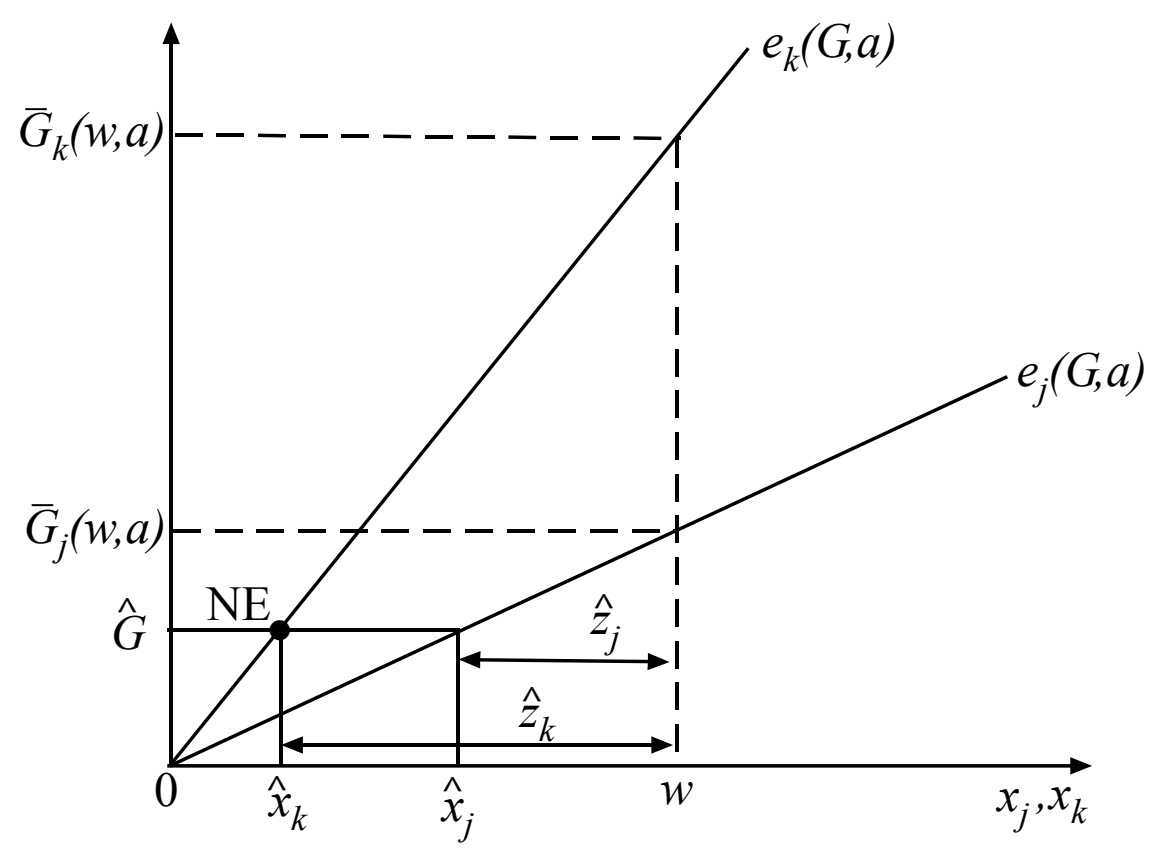

Figure 2. Exploitation based on taste differences

Case 3: All agents have the same income $w$ and the same utility function $u\left(x_{i}, G\right)$ but different productivity parameters $a_{i}$.

We assume that agents are ranked according to their productivities, i.e., $a_{1} \leq \ldots \leq a_{n}$. Then we have for the expansion paths originating from the common utility function that $e\left(G, a_{j}\right) \geq e\left(G, a_{k}\right)$ holds at each $G$ if $j<k$ and thus $\bar{G}_{1}\left(w, a_{1}\right) \leq \ldots \leq \bar{G}_{n}\left(w, a_{n}\right)$. This can be depicted in a diagram that is completely analogous to Figure 2 .

By the same argument as in cases 1 and 2 above, it now follows that, at the NE, agents with a greater public good productivity, ceteris paribus, contribute more to the public good than agents with a lower productivity. When agents $j$ and $k$ make positive contributions to the public good, we have hence $\hat{z}_{j}=w-e\left(\hat{G}, a_{j}\right) \leq w-e\left(\hat{G}, a_{k}\right)=\hat{z}_{k}$.

In case 3 , all non-contributors' utility $u(w, \hat{G})$ is the same at the NE, while contributors' utility $u\left(\hat{x}_{i}, \hat{G}\right)$ is smaller, the greater her public good productivity. This reflects the "curse of being more productive" in voluntary public good provision, which - in a somewhat different context - has also been observed by Buchholz and Konrad (1994) and Ihori (1996). 


\section{Interaction of the Partial Effects}

The partial effects that underlie the above three cases can offset each other. Either due to weak preferences for the public good or a low public good productivity, it is possible that an agent with a relatively high income contributes less to the public good than a poorer one. The interaction of the different partial effects can be visualized by the following example, in which we consider an economy with two agents $i=1,2$. Agent 1 has the initial endowment $w_{1}=1$, the symmetric Cobb-Douglas utility function $u_{1}\left(x_{1}, G\right)=x_{1} G$ and the productivity parameter $a_{1}=1$. Agent 2 has a higher initial endowment, assumed to be $w_{2}=2$. Concerning agent 2's further characteristics, we distinguish two cases:

- Agent 2 has the same productivity parameter as agent 1 , i.e. $a_{2}=1$, but possesses a different asymmetric Cobb-Douglas utility function $u_{2}\left(x_{2}, G\right)=x_{1} G^{\frac{1}{2}}$. Then, at each $(x, G)$, we have $m r s_{2}(x, G)=2 \frac{G}{x}>\frac{G}{x}=m r s_{1}(G, x)$, i.e. agent 2 has a weaker preference for the public good than agent 1 according to the definition given above. The Nash equilibrium is given by $\hat{x}_{1}=\hat{G}=\frac{3}{4}$ and $\hat{x}_{2}=\frac{3}{2}$. The public good contributions of the two countries are thus $\hat{z}_{1}=\frac{1}{4}$ and $\hat{z}_{2}=\frac{1}{2}$, respectively, so that both countries - despite their different endowments - spend the same share of their initial endowment on the public good, i.e., $\frac{\hat{z}_{1}}{w_{1}}=\frac{\frac{1}{4}}{1}=\frac{\frac{1}{2}}{2}=\frac{\hat{z}_{2}}{w_{2}}$.

- Agent 2 has the same utility function as agent 1 , i.e. $u_{1}\left(x_{2}, G\right)=x_{2} G$, but possesses a smaller public good productivity $a_{2}=\frac{1}{2}$. The Nash equilibrium then is given by $\hat{x}_{1}=\hat{G}=\frac{2}{3}$ and $\hat{x}_{2}=\frac{4}{3}$, which gives $\hat{z}_{1}=\frac{1}{3}$ and $\hat{z}_{2}=\frac{2}{3}$. Again, the relative income shares of public good expenditures are identical in both countries, i.e. $\frac{\hat{z}_{1}}{w_{1}}=\frac{\frac{1}{3}}{1}=\frac{\frac{2}{3}}{2}=\frac{\hat{z}_{2}}{w_{2}}$.

These two examples show how the conventional exploitation effect may be partly or wholly counterbalanced by the effects that underlie the other two versions of the exploitation maxim presented in this paper. 


\section{Some Empirical Applications}

To highlight the relevancy of the three exploitation hypotheses, we provide some illustrations drawn from the international public goods.

The first application of the classical exploitation hypothesis was to the NATO alliance, in which - despite the member states' commitment to mutual assistance - the national contributions to collective defence essentially are made on a voluntary basis. With respect to burden sharing within the NATO, Olson and Zeckhauser (1966) hypothesized that rich allies would shoulder the alliance defence burdens for the poor allies, where gross domestic product (GDP) served as a proxy for income. To test this exploitation hypothesis, they used the allies' military expenditure (ME) as share of GDP as the burden measure. Using a Spearman rank correlation test, Olson and Zeckhauser (1966) found a significant positive relationship for 1964 between the allies' ME/GDP ranks and their GDP ranks, thus supporting the income-based exploitation hypothesis in case 1.

In more recent times, Greece and Turkey spend a greater proportion of their GDP on defence than do their richer allies in NATO (Sandler and Murdoch, 2000). For example, in 2000, Greece and Turkey devoted 3.6\% and 3.7\% of their GDP to military expenditure, respectively, while, e.g., the United Kingdom spent 2.4\% and Germany only $1.5 \%$ of GDP on ME (SIPRI 2015). Clearly, grievances between Greece and Turkey raised their preference for defence and motivated their greater burdens compared to their much richer allies in NATO, which agrees with case 2. Even though the ME/GDP ratio of these two countries has fallen since then it is still higher than for most of the other NATO members.

For now, remarkably, the ME/GDP ratio is also higher for some new NATO members in Eastern Europe, which - from historical reasons - perceive a stronger foreign threat. So in 2014 - according to the World Bank - it has been 1.9\% both in Estonia and Poland and even $2.7 \%$ in Lithunia. Also for the permanent Western members of the UN Security Council (United States, United Kingdom and France), per capita military expenditures are relatively high because these countries are feeling responsible for global security far beyond their own borders and that of their allies.

To further illustrate case 2 and also consider case 3 in the context of defence economics, we consider the US-Israeli alliance using data from the Stockholm International Peace Research Institute (SIPRI) (2015). Even though Israel has a much smaller GDP than the United States, Israel's ME/GDP exceeded that of the United States as follows: $23 \%$ compared to $7.7 \%$ in $1970,13.7 \%$ compared to $5.3 \%$ in 1990 , and $5.2 \%$ compared to $3.5 \%$ in 2014 (SIPRI, 2015). Obviously, Israel has a much greater preference for de- 
fence insofar as it is surrounded by enemy states, all of which have fought wars with Israel. Moreover, the productivity of Israel in defending itself in the region is greater than that of the United States, which must project its power to the Middle East, meaning reduced productivity from their respective arsenal in meeting the specific threats in this region. Also, Israel has adapted its weapon systems, even those purchased from the United States, to be suited to battlefields surrounding Israel. Thus, case 3 also comes into play.

Other examples of case 2 exist. Consider peacekeeping as a pure public good that brings stability to the world. In 2000, Denmark devoted the largest share of its GDP to peacekeeping of the NATO allies to UN and non-UN-led peacekeeping missions even though its GDP was ranked $13^{\text {th }}$ of the 18 allies (Shimizu and Sandler, 2003). The other three highest ranked contributors in 2000 were the Netherlands, Italy, and Norway. Clearly some poorer countries - mainly due to specific political attitudes and power relations within these countries - displayed a greater preference for providing peacekeeping as a public good.

Another field of application of our theoretical results is foreign aid. Given Sweden's, Denmark's, and Norway's stated commitment to foreign aid, they provide a much greater share of their GDP to helping poor countries than rich countries, such as the United States (Sandler, 2004). Foreign aid is a public good providing non-excludable and nonrival benefit to countries that care about less fortunate countries. The Scandinavian contributions to foreign aid abide by case 2 .

Addressing cross-border pollution can also represent cases 2 and 3 . In the case of switching from ozone-shield-depleting substances, advanced countries, such as the United States, Japan, and many EU countries, had the edge over other countries. This advantage was so great that - according to our case 2 - the Montreal Protocol not only required higher abatement efforts from these countries but even the setting up of a Multilateral Fund to assist less capable countries abide by the treaty at the expense of the rich countries (Sandler, 2004). Productivity differences with respect to the reduction of sulphur emissions also meant that the Oslo Protocol required greater percentage cutbacks from those countries with a greater productivity in mitigation measures (Finus and Tjøtta, 2003). Both of these examples result in an exploitation of the greater productive country for the betterment of everyone in international environmental agreements. In this context it is important to note that these agreements are considered as mere 
manifestations of abatement efforts, which countries would have undertaken on a voluntary basis (Murdoch and Sandler, 1997).

In the topical case of climate change, Germany - by virtue of a sense of environmental morality (a relatively strong position of the Green Party in its political system) - has been more ambitious in greenhouse gas mitigation than the United States even though its GDP is lower, which reflects case 2. Germany's strong preference for climate protection particularly shows up in its enormous subsidies for renewable energies, which totalled about 24 billion Euros in 2015.

Productivity differences - as in case 3 - also characterize efforts to reduce climate change. High-abatement-cost countries are typically reluctant to become active in climate policy. This, e.g., is the case for countries (such as India, China and, among the European countries, especially Poland) whose electricity supply heavily depends on the use of coal, which is abundantly available at low cost in these countries so that the opportunity costs of turning to a climate-friendly energy supply would be high for them. That abatement costs may affect a country's contribution to global climate protection is also corroborated by the recent experience in the United States: Progress in fracking technology and falling costs of shale gas production made it possible to substitute less carbon-intensive natural gas for dirty coal. This is considered as a major cause for the decrease of greenhouse gas emissions in the United States, which fell by $9 \%$ between 2005 and 2013 (EPA, 2015).

Moreover, some countries have a productive advantage in terms of the use of renewable energy sources. In the United States, Texas, Hawaii, and Wyoming have an advantage over many other states in the production of wind power owing to wind patterns, while coastal states will have an advantage in wave-produced power as the technology is further developed. Sun-drenched states, such as Arizona, Nevada, and Texas have a potential advantage in the production of solar energy. In Europe, e.g., Denmark and Northern Germany have an advantage in wind power, whereas the southern European countries (such as Spain, Italy, and Greece) have lower costs in generating solar energy. One might expect that these comparative advantages will foster contributions to the global public good climate protection. 
Acknowledgments: Wolfgang Buchholz gratefully acknowledges financial support from the German Federal Ministry of Education and Research BMBF through the research project ECCUITY (FKZ 01LA1104B) as part of the funding priority "Economics of Climate Change". Todd Sandler received support from the Vibhooti Shukla endowment at the University of Texas at Dallas.

\section{References}

Andreoni, J. (1988) Privately provided public goods in a large economy: the limits of altruism. Journal of Public Economics, 35(1), 57-73.

Andreoni, J., McGuire, M. C. (1993) Identifying the free riders: a simple algorithm for determining who will contribute to a public good. Journal of Public Economics, 51(3), 447-454.

Bergstrom, T. C., Blume, L., Varian, H. (1986) On the private provision of public goods. Journal of Public Economics, 29(1), 25-49.

Boadway, R., Hayashi, M. (1999) Country size and the voluntary provision of international public goods. European Journal of Political Economy, 15(4), 619-638.

Buchholz, W., Konrad, K. A. (1994) Global environmental problems and the strategic choice of technology. Journal of Economics, 60(3), 299-321.

Cornes, R., Hartley, R. (2007) Aggregative public good games. Journal of Public Economic Theory, 9(2), 201-219.

Cornes, R., Sandler, T. (1996) The Theory of Externalities, Public Goods, and Club Goods, $2^{\text {nd }}$ Edition. Cambridge, Cambridge University Press.

Environmental Protection Agency (EPA) (2015) Climate change indicators in the United States: U.S. greenhouse gas emissions. Updated June 2015. Washington D.C.

Finus, M., Tjøtta, S. (2003) Oslo Protocol and sulphur reduction: the great leap forward. Journal of Public Economics, 87(9-10), 2031-2048.

Fraser, C. D. (1992) The uniqueness of Nash equilibrium in the private provision of public goods. Journal of Public Economics, 49(3), 389-390.

Ihori, T. (1996) International public goods and contribution productivity differentials. Journal of Public Economics, 61(1), 139-154.

Ihori, T., McGuire, M. C., Nakagawa, S. (2014) International security, multiple public good provision, and the exploitation hypothesis. Defence and Peace Economics, 25(3), 213-229.

Murdoch, J., Sandler, T. (1997) The voluntary provision of a pure public good: the case of reduced CFC emissions and the Montreal Protocol. Journal of Public Economics, 63(2), 331-349. 
Olson, M. (1965) The Logic of Collective Action. Cambridge, MA, Havard University Press.

Olson, M., Zeckhauser, R. (1966) An economic theory of alliances. Review of Economics and Statistics, 48(3), 266-279.

Sandler, T. (1992) Collective Action: Theory and Applications. Ann Arbor, University of Michigan Press.

Sandler, T. (2004) Global Collective Action. Cambridge, Cambridge University Press

Sandler, T. (2015) Collective action: fifty years later. Public Choice, 164(3-4), 195-216.

Sandler, T., Murdoch, J. C. (2000) On sharing NATO defence burdens in the 1990s and beyond. Fiscal Studies, 21(3), 297-327.

Shimizu, H., Sandler, T. (2003) NATO peacekeeping and burden sharing, 1994-2000. Public Finance Review, 31(2), 123-143.

Stockholm International Peace Research Institute (SIPRI) (2015) SIPRI extended military expenditure database, preliminary version. Given by SIPRI to authors. 